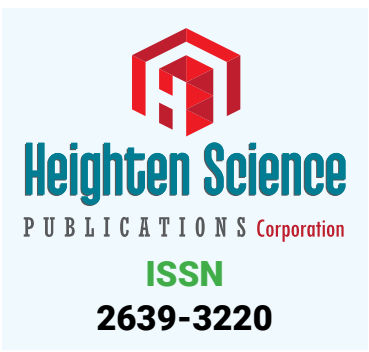

*Address for Correspondence: Jes Paul, Department of Molecular and Cellular Physiology, Albany Medical College, Albany, NY, USA, Tel: 4049802103; Email: paulj5@amc.edu; jesrejoice@gmail.com

Submitted: 12 June 2018

Approved: 28 June 2018

Published: 29 June 2018

Copyright: @2018 Paul J. This is an open access article distributed under the Creative Commons Attribution License, which permits unrestricted use, distribution, and reproduction in any medium, provided the original work is properly cited.

\section{Discrepancy in Microglia and Peripheral Monocytic Cells - A scope in the Pathophysiology of Psychiatric maladies}

\author{
Jes Paul* \\ Department of Molecular and Cellular Physiology, Albany Medical College, Albany, NY, USA
}

\section{Abstract}

Broad medleys of research have recognized the microglial activation in perilous psychiatric maladies such as schizophrenia, bipolar disorder, and major depressive disorder. There is a scenario of enlivening of peripheral monocytic cells along with the microglial interactions within the body while considering the Pathogenesis of psychiatric disorders. this review, epitomize and discuss the activation of microglia and monocytic cells in psychiatric disorders, thereby showcasing the potential association between these cell types and the Pathogenisis of the ailment, and proffer perspectives for future research on these processes.

\section{Introduction}

In a variation of psychiatric disorders suchlike schizophrenia, bipolar disorder and major depressive disorder, a diversity of postmortem brain studies and recent positron emission tomography (PET)-based studies indicated an association between alterations in monocytic features and psychiatric disorders [1-3].

In the course of pathological conditions, monocytes are initiated from peripheral blood into the brain, where they collaborate with microglia in immune responses [4-6]. Peripheral monocytes have the capability to set apart into macrophages and dendritic cells in peripheral tissues, which share resemblance with microglia in their cellular morphology and roles, such as phagocytic activities, uniform gene expression profiles and the locution of cell surface markers and cytokine genesis [4-7]. This review airs the potential mechanisms linking microglial and monocytic activation with the pathogenesis of psychiatric disorders. Also it portrays and propose directions for future research on these potential associations.

\section{Recent findings}

Numerous Postmortem brain studies have unveiled the link between psychiatric disorders and microglial activation [8-12]. Activated microglial cells were speculated to be increased in prefrontal white matter from patients with schizophrenia, but not that from patients with bipolar disorder while the qualitative assessment of microglial morphology is done $[8,11]$. Disclosed that in immunostaining studies the expression of Human Leukocyte Antigen-antigen D Related (HLA-DR), which reacts with activated microglia, was inflated in the frontal cortices of patients with schizophrenia. antigenpresenting cells) was increased in the white matter of patients with schizophrenia as per Fillman et al. [10]. Positive correlations such as changes in the expression of genes that encode markers of activated microglia are seen among the several activated microglial markers in subjects with schizophrenia [9].

How to cite this article: Paul J. Discrepancy in Microglia and Peripheral Monocytic Cells - A scope in the Pathophysiology of Psychiatric maladies. J Neurosci Neurol Disord. 2018; 2: 028-032. https://doi.org/10.29328/journal.jnnd.1001011 


\section{Pathogenesis of psychiatric chaos}

Microglial Function and Potential Mechanisms is the mess in Psychiatric Upset. Hematopoietic stem cells in the yolk sac in the early developmental stage is the genesis of Microglia which comprise $\sim 12 \%$ of cells in the central nervous system (CNS); these cells are not analogously dispersed [7]. More microglia are sited close to neurons in the gray matter, with the peaked concentrations in the hippocampus, olfactory telencephalon, basal ganglia, and substantia nigra.

Pile of evidence from fate-mapping studies allude to the origin of most microglia is not the bone marrow after birth but hematopoietic stem cells in the yolk sac in the early developmental stage [6,13-15]. Novel transgenic approaches have shown clear differences in the cellular characteristics of microglia and macrophages in the brain [16-18]. In contrast to macrophages, microglia are long-lived and are not replaced by circulating peripheral monocytes under physiological conditions. By various pathological conditions, such as trauma, infection, or other damage to brain tissue Activated microglia retract their cellular processes and transform from a ramified state into an ameboid morphology, Activated microglial functions include phagocytosis and the fabrica1tion and discharge of cytokines, reactive oxygen species and nitrogen species [19-21].

Potential pathogenic mechanisms underlying the involvement of peripheral monocytes in psychiatric disorders

Being the precursors of tissue macrophages, osteoclasts, and antigen-presenting cells the Monocytes are differentiated in to five subsets based on the different surface markers [4,22,23]. Almost half of them corresponds to subset 1 and are characterized by surface marker profiles with abundant CD14 and a lack of CD16 expression .Both subsets 2 and 3 have CD16 expression and comprise active phagocytic cells in common but differs in the expression of CD14. Increased expression of CD14 is seen in subset 2 compared with subset 3. Subset 4 is a precursor of dendritic cells with high expression of CD40. While Subset 5, the smallest subset, even though it shares many surface markers with subset 1 ; however, it differs in the additional expression of CD56, a marker of immature monocytes. Conversation between subsets among peripheral monocytes can arise concomitant with the demarcation of microglia in the brain under certain pathogenic conditions allied to psychiatric diseases, although these potentialities remain to be clarified.

Virtual mechanisms linking arousal of microglial and peripheral monocytic cells to psychiatric disorder

Parallel responses are manifested by both microglia and monocytes to systemic stimuli. These two cell types evince alike profiles for cytokine production, such as interleukin (IL) $1 \beta$, IL-6, IL-8 or tumor necrosis factor (TNF)- $\alpha$. They also show likeness in surface markers, such as cluster of differentiation 14 (CD14), major histocompatibility complex (MHC) molecules, and chemokine receptors. In response to Local stimuli in the brain, the circulating monocyte-derived macrophage/dendritic cells are pledged into the central nervous system (CNS). Immigrant monocytic cells, which have concise life span may collude with microglia in the innate immune response. Signals are transduced between microglia and circulating monocytes via the blood-brain barrier (BBB). Biological reactions are advertised through neuronal signal transduction. Microglia and peripheral monocytic cells may also wield related Peripheral inflammation.

Reaction of microglia and peripheral monocytic cells to stimuli (Endogenous or Exogenous)

As cited in the introduction, microglia and monocytes have concordant purpose such as phagocytosis and the discharge of pro-inflammatory cytokines, as well as 
parallel expression of surface markers, such as CD14, MHC molecules, and chemokine receptors [4-7]. Schmitz et al. [7], detected over-representation of genes connected with Alzheimer's disease among the expression profiles specific to microglia and monocytes [24]. All these studies when taken together, it bespeaks that microglia and monocytes have harmonious gene expression pattern under normal conditions; however, there occur changes in the pattern of countenance profiles in response to stimuli are quite different.

\section{Neuropsychoimmune interactions in the pathophysiology of psychiatric disorders (Innate Immune Response)}

Detriment to the CNS (central nervous system) habitually ends in the enrollment of circulating immune cells, including monocytes, which ensue in an innate immune response that consists of microglia and monocyte-derived macrophages/dendritic cells [6]. In disorders like Alzheimer's disease, triggered microglia have been kindred with amyloid- $\beta$-induced neurotoxicity, and microglia were also injured by amyloid species $[6,25,26]$. Likewise the rectification from spinal cord injury in mice has been reported to rest more on infiltrating monocyte-derived macrophages than on resident microglia.

\section{Interactions through the blood-brain barrier and synergy through neuronal networking}

Synergy between microglia and monocytes are governed by cytokines. Even though both microglia and monocytes are actuated by and exempt pro-inflammatory cytokines, such as IL-1 $\beta$, IL-6, IL-8 or TNF- $\alpha[4,7]$, as the cytokines are relatively large molecules and rarely cross the blood-brain barrier (BBB). But there are some agencies and routes by which they can cross the barrier, such as variances in the barrier's permeability, crossway though circumventricular organs, or the use of precise transporters or receptors [27].

Moreover, many Studies have proclaimed that these cytokines are in peak level in the blood of psychiatric patients. Therefore, it is very much logical to suspect that these cytokine activation might underlie interplays between microglia and monocytes. Diverse discoveries imply that mild peripheral inflammation, which bestows to the pathogenesis of psychiatric disease-related events, including fatigue, may instigate microglial activation through neuronal networking.

In addition to that, the symbioses between microglia and neuronal networks inflect myeloid cell proliferation through the autonomic nervous system $[28,29]$.

\section{Conclusion}

This work focus to identify and characterize novel targets that are potentially involved in the pathophysiology of psychiatric disorders. Congruous Microglia and monocytes have similar functions, surface markers, and gene expression profiles. Both cell types manumit pro-inflammatory cytokines when switched on in response to stimuli in the brain under various pathological conditions. Agglomerating data also betoken interactions between monocytes and microglia. New strategies can be expounded by doing Research into these interactions that may lead to the pathogenesis of psychiatric diseases. These interactions and chemistries can be used as peripheral biomarkers that echo the pathological conditions in the brain, conjunction with microglial activation related to the headway or Inflection of disease.

\section{Conflict of interest statement}

The research was conducted in the absence of any commercial or financial relationships that could be construed as a potential conflict of interest. 


\section{References}

1. Theodoropoulou S, Spanakos G, Baxevanis CN, Economou M, Gritzapis AD, et al. Cytokine serum levels, autologous mixed lymphocyte reaction and surface marker analysis in never medicated and chronically medicated schizophrenic patients. Schizophr Res. 2001: 47: 13-25. Ref.: https://tinyurl.com/yc8hn2g5

2. Padmos RC, Hillegers MH, Knijff EM, Vonk R, Bouvy A, et al. A discriminating messenger RNA signature for bipolar disorder formed by an aberrant expression of inflammatory genes in monocytes. Arch Gen Psychiatry. 2008; 65: 395-407. Ref.: https://tinyurl.com/ycuy8zew

3. Drexhage RC, Hoogenboezem TH, Versnel MA, Berghout A, Nolen WA, et al. The activation of monocyte and T cell networks in patients with bipolar disorder. Brain Behav Immun. 2011; 25: 12061213. Ref.: https://tinyurl.com/yc8kq6zp

4. Beumer W, Gibney SM, Drexhage RC, Pont-Lezica L, Doorduin J, et al. The immune theory of psychiatric diseases: a key role for activated microglia and circulating monocytes. J Leukoc Biol. 2012; 92: 959975. Ref.: https://tinyurl.com/ybb4oux3

5. Shechter R, Schwartz M. Harnessing monocyte-derived macrophages to control central nervous system pathologies: no longer 'if' but 'how'. J Pathol. 2013; 229: 332-346. Ref.: https://tinyurl.com/y7lzabt

6. Prinz $\mathrm{M}$, Priller J. Microglia and brain macrophages in the molecular age: from origin to neuropsychiatric disease. Nat Rev Neurosci. 2014; 15: 300-312. Ref.: https://tinyurl.com/ybkufv35

7. Schmitz G, Leuthäuser-Jaschinski K, Orsó E. Are circulating monocytes as microglia orthologues appropriate biomarker targets for neuronal diseases? Cent Nerv Syst Agents Med Chem. 2009; 9: 307-330. Ref.: https://tinyurl.com/y7xnsauh

8. Radewicz K, Garey LJ, Gentleman SM, Reynolds R. Increase in HLA-DR immunoreactive microglia in frontal and temporal cortex of chronic schizophrenics. J Neuropathol Exp Neurol. 2000; 59: 137-150. Ref.: https://tinyurl.com/y8y2m558

9. Tang B, Capitao C, Dean B, Thomas EA. Differential age- and disease-related effects on the expression of genes related to the arachidonic acid signaling pathway in schizophrenia. Psychiatry Res. 2012 196: 201-206. Ref.: https://tinyurl.com/yd9ebusa

10. Fillman SG, Cloonan N, Catts VS, Miller LC, Wong J, et al. Increased inflammatory markers identified in the dorsolateral prefrontal cortex of individuals with schizophrenia. Mol Psychiatry. 2013; 18: 206214. Ref.: https://tinyurl.com/y7vvjpv3

11. Hercher C, Chopra V, Beasley CL. Evidence for morphological alterations in prefrontal white matter glia in schizophrenia and bipolar disorder. J Psychiatry Neurosci. 2014; 39: 376-385. Ref.: https://tinyurl.com/yckzl89r

12. Torres-Platas SG, Cruceanu C, Chen GG, Turecki G, Mechawar N. Evidence for increased microglial priming and macrophage recruitment in the dorsal anterior cingulate white matter of depressed suicides. Brain Behav Immun. 2014; 42: 50-59. Ref.: https://tinyurl.com/yaqhkzae

13. Ginhoux F, Greter $M$, Leboeuf $M$, Nandi $S$, See $P$, et al. Fate mapping analysis reveals that adult microglia derive from primitive macrophages. Science. 2010; 330: 841-845. Ref.: https://tinyurl.com/ycq4wtnl

14. Schulz C, Gomez Perdiguero E, Chorro L, Szabo-Rogers H, Cagnard N, et al. A lineage of myeloid cells independent of Myb and hematopoietic stem cells. Science. 2012; 336: 86-90. Ref.: https://tinyurl.com/ycwl9vmk

15. Kierdorf K, Erny D, Goldmann T, Sander V, Schulz C, et al. Microglia emerge from erythromyeloid precursors via Pu.1- and Irf8-dependent pathways. Nat Neurosci. 2013; 16: 273-280. Ref.: https://tinyurl.com/yaarr2ta

16. Goldmann $T$, Wieghofer $P$, Muller PF, Wolf $Y$, Varol $D$, et al. A new type of microglia gene targeting shows TAK1 to be pivotal in CNS autoimmune inflammation. Nat Neurosci. 2013; 16: 1618-1626. Ref.: https://tinyurl.com/y8d2xeom

17. Parkhurst CN, Yang G, Ninan I, Savas JN, Yates JR, et al. Microglia promote learning-dependent synapse formation through brain-derived neurotrophic factor. Cell. 2013; 155: 1596-1609. Ref.: https://tinyurl.com/y982squc

18. Yona S, Kim KW, Wolf $Y$, Mildner A, Varol D, et al. Fate mapping reveals origins and dynamics of monocytes and tissue macrophages under homeostasis. Immunity. 2013; 38: 79-91. Ref.: https://tinyurl.com/ya69vcpm

19. Barger SW, Basile AS. Activation of microglia by secreted amyloid precursor protein evokes release of glutamate by cystine exchange and attenuates synaptic function. J Neurochem. 2001; 76: 846854. Ref.: https://tinyurl.com/yb2fk4yu 
20. Takaki J, Fujimori K, Miura M, Suzuki T, Sekino $Y$, et al. L-glutamate released from activated microglia downregulates astrocytic L-glutamate transporter expression in neuroinflammation: the 'collusion'hypothesis for increased extracellular L-glutamate concentration in neuroinflammation. $J$ Neuroinflammation. 2012; 9: 275. Ref.: https://tinyurl.com/ycljbm2s

21. Réus GZ, Fries GR, Stertz L, Badawy M, Passos IC, et al. The role of inflammation and microglial activation in the pathophysiology of psychiatric disorders. Neuroscience. 2015; 300: 141-154. Ref.: https://tinyurl.com/yakqh2dr

22. Schmitz G, GrandI M. Role of redox regulation and lipid rafts in macrophages during Ox-LDL-mediated foam cell formation. Antioxid Redox Signal. 2007; 9: 1499-1518. Ref.: https://tinyurl.com/y9vf3foz

23. Gordon S, Taylor PR. Monocyte and macrophage heterogeneity. Nat Rev Immunol. 2005; 5: 953-964. Ref.: https://tinyurl.com/y9eelb4a

24. Lutter D, Ugocsai P, Grandl M, Orso E, Theis F, et al. Analyzing M-CSF dependent monocyte/ macrophage differentiation: expression modes and meta-modes derived from an independent component analysis. BMC Bioinformatics. 2008; 9: 100. Ref.: https://tinyurl.com/ydbsblcj

25. Simard AR, Soulet D, Gowing G, Julien JP, Rivest S. Bone marrow-derived microglia play a critical role in restricting senile plaque formation in Alzheimer's disease. Neuron. 2006; 49: 489-502. Ref.: https://tinyurl.com/ybr2rxb5

26. Mildner A, Schlevogt B, Kierdorf K, Böttcher C, Erny D, et al. Distinct and non-redundant roles of microglia and myeloid subsets in mouse models of Alzheimer's disease. J Neurosci. 2011; 31: 11159-11171. Ref.: https://tinyurl.com/y9sggxqk

27. Banks WA, Erickson MA. The blood-brain barrier and immune function and dysfunction. Neurobiol Dis. 2010; 37: 26-32. Ref.: https://tinyurl.com/ya68yfzp

28. Spiegel A, Kalinkovich A, Shivtiel S, Kollet O, Lapidot T. Stem cell regulation via dynamic interactions of the nervous and immune systems with the microenvironment. Cell Stem Cell. 2008; 3: 484-492. Ref.: https://tinyurl.com/y8y7vabf

29. Jes Paul, Paulose CS, John PS, Sreekanth R, Mathew Philip, et al. Spinal Cord Regeneration and Functional Recovery: Neurotransmitter's Combination and Bone Marrow Cells Supplementation. J Current Sci. 2009; 25: 546-549. 